\title{
Measuring Cognitive Workload with Low-Cost Electroencephalograph
}

\author{
Avi Knoll, Yang Wang, Fang Chen, Jie Xu, \\ Natalie Ruiz, Julien Epps, and Pega Zarjam \\ National ICT Australia \\ University of New South Wales \\ \{yang.wang, fang.chen, natalie.ruiz, \\ julien.epps, pega.zarjam\}@nicta.com.au \\ ajk@cse.unsw.edu.au, jie.jackxu@gmail.com
}

\begin{abstract}
Electroencephalography (EEG) is an important physiological index of cognitive workload. While previous research has employed high-end EEG devices, this work investigates the feasibility of measuring cognitive workload with a low-cost EEG system. In our experiment, EEG signals are recorded from subjects performing silent reading tasks under different difficulty levels. Experimental results demonstrate the effectiveness of cognitive workload evaluation even with low-cost EEG equipment.
\end{abstract}

Keywords: Cognitive workload, electroencephalography (EEG), physiological index.

\section{Introduction}

In recent years, research efforts have been geared towards measuring human mental states such as cognitive workload and task engagement. Cognitive workload refers to the amount of mental demand imposed by a particular task on a person [3]. Measuring cognitive workload is an important issue in various research and application areas of human-computer interaction, as it can be utilized to evaluate the efficacy of interfaces and build adaptive interaction systems. With the advance of modern sensing technologies, a variety of physiological measures have been developed for the assessment of cognitive workload. Among these techniques, electroencephalography (EEG) has become a popular physiological index that allows continuous monitoring of subjects' cognitive workload in a convenient way.

Previous research has demonstrated that EEG signals are sensitive to cognitive load changes in various tasks [1]. Gevins and Smith [5] demonstrated that spectral features of the theta and alpha frequency bands correlate with task difficulty levels in simulated flight tasks and n-back tests. Fitzgibbon et al. [4] also found that the power of gamma band could be augmented by various cognitive tasks. Berka et al. [2] employed discriminant function analysis on spectral features for monitoring cognitive workload and task engagement in different tasks including digit span, mental arithmetic, image learning and memory tests. Grimes et al. [6] and Zarjam et al. [9] investigated EEG based classification and evaluation of subjects' working memory 
load. A feature selection scheme based on mutual information was proposed in [6] to deal with physiological drift. EEG has also been used to monitor cognitive workload in various military tasks under complex environments [8].

Although EEG is a promising tool for continuous measurement of cognitive workload, most previous research has employed high-end EEG systems costing more than $\$ 15,000$ (e.g. see www.biosemi.com), which limits their widespread usage in human-computer interfaces. On the other hand, low-cost (under \$1000) EEG headsets have become accessible for HCI research in recent years [7]. This work takes an initial step in exploring the feasibility of cognitive workload evaluation using a lowcost multi-channel EEG system.

\section{Experiment}

Sixteen students and employees (16-46 years old, 4 females) were invited to perform silent reading tasks. Brain waves from each subject were recorded with a low-cost EEG device originally designed for gaming interfaces (Emotiv EPOC, a 14 channel $128 \mathrm{~Hz}$ neuro-signal acquisition and processing wireless neuroheadset [10], see Figure 1). Channel names based on the International 10-20 locations are: AF3, F7, F3, FC5, T7, P7, O1, O2, P8, T8, FC6, F4, F8, AF4. During the experiment, each subject was asked to silently read the text displayed on-screen, with a viewing distance of $70 \mathrm{~cm}$ (see Figure 2). Similar to [9], different task difficulty levels were employed to manipulate cognitive workload during the experiment.
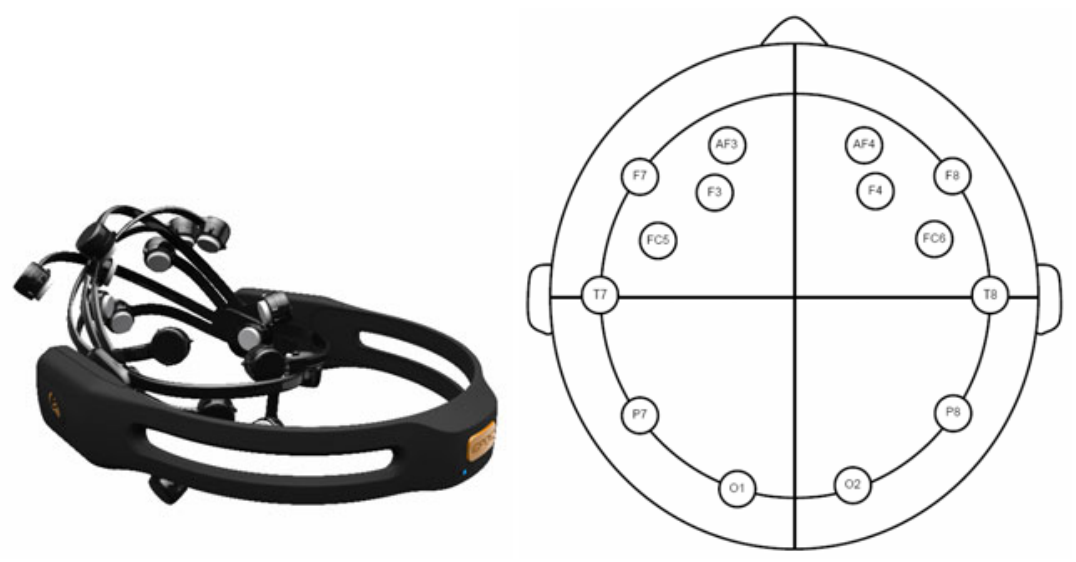

Fig. 1. A low-cost EEG device (Eomtiv EPOC neuroheadset [10]) and layout of EEG channels

There were three levels of task difficulty in total: low (level 1), medium (level 2) and high (level 3). For each difficulty level, 4 text pages were sequentially displayed on the screen, with each page appearing for 30 seconds. In the low level task, the subject was required to press the left mouse button when he encountered any 3 letter word during silent reading. In the medium level task, the subject was required to press 
the left or middle button respectively, each time he encountered either a 3 or a 4 letter word. Likewise, in the high level task, the subject was required to press the either the left, middle, or right button when he saw a 3, 4, or 5 letter word respectively. The task difficulty levels were administered in a randomized fashion. There was a 30 second resting period after the task for each difficulty level. One minute baseline data (with the subject looking at a blank screen) was recorded at both the beginning and the end of the whole experiment for each subject. The subject was asked to refrain from eye blinking and to stay as still as possible during the baseline period and task period. However, the subject was free to blink and move their head naturally during each rest period.

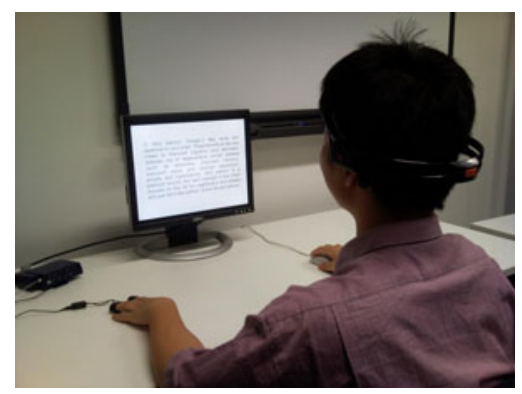

Fig. 2. Experiment setup

\section{Analysis}

The EEG signals were first divided into segments of 1.5 seconds in length. Statistical features including mean, variance, root mean square (RMS), spectral powers of theta (3-7 Hz), alpha $(8-12 \mathrm{~Hz})$, beta $(13-29 \mathrm{~Hz})$, and gamma $(30+\mathrm{Hz})$ frequency bands were then calculated for each data segment.

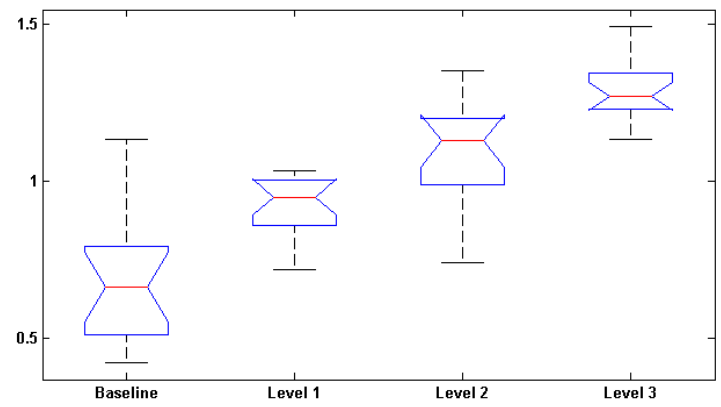

Fig. 3. Box plot of normalized RMS values (sample minimum, lower quartile, median, upper quartile, and maximum) from nodes F3 and F4 at different task difficulty levels 
Among the features obtained from different EEG channels, RMS from nodes F3 and F4 exhibit significant correlation with task difficulty $(\mathrm{F}>38, \mathrm{p}<0.01$ in ANOVA test). This finding is consistent with previous research indicating that the brain frontal lobes play an important role in cognitive tasks associated with attention and mental effort [5]. Figure 3 plots the distribution of normalized RMS acquired from the two frontal channels at different workload levels for all the subjects. It can be seen that the feature value consistently increases when the task difficulty level is increased.

Moreover, the spectral power of gamma frequency band at nodes AF3 and AF4 shows a statistically significant difference between the baseline condition and task condition ( $\mathrm{F}>28, \mathrm{p}<0.01$ in ANOVA test), which is consistent with previous study on gamma activation of EEG during cognitive tasks [4]. There is an increase in average gamma power with each rise in task difficulty. However, the difference between task levels is not statistically significant $(\mathrm{p}>0.05)$.

\section{Conclusion}

This work investigates the feasibility of cognitive workload evaluation using a lowcost EEG system. It is demonstrated that cognitive workload could be effectively measured even with low-cost electroencephalograph. The experimental results are consistent with previous research on cognitive workload. We hope that this work will promote the application of EEG-based physiological measures in various HCI areas involving cognitive workload evaluation.

\section{References}

1. Antonenko, P., Paas, F., Grabner, R., van Gog, T.: Using electroencepahlography to measure cognitive load. Educational Psychology Review 22, 424-438 (2010)

2. Berka, C., Levendowski, D., Lumicao, M., Yau, A., Davis, G., Zivkovic, V., Olmstead, R., Tremoulet, P., Craven, P.: EEG correlates of task engagement and mental workload in vigilance, learning, and memory tasks. Aviation, Space, and Environmental Medicine 78, B231-B244 (2007)

3. Cain, B.: A review of the mental workload literature. Technical Report, Defence Research and Development Canada Toronto (2007)

4. Fitzgibbon, S., Pope, K., Mackenzie, L., Clark, C., Willoughby, J.: Cognitive tasks augment gamma EEG power. Clinical Neurophysiology 115, 1802-1809 (2004)

5. Gevins, A., Smith, M.: Neurophysiological measures of cognitive workload during humancomputer interaction. Theoretical Issues in Ergonomic Science 4, 113-131 (2003)

6. Grimes, D., Tan, D., Hudson, S., Shenoy, P., Rao, R.: Feasibility and pragmatics of classifying working memory load with an electroencephalograph. In: Proc. ACM CHI Conf. Human Factors in Computing Systems, pp. 835-844 (2008)

7. Lee, J., Tan, D.: Using a low-cost electroencephalograph for task classification in HCI research. In: Proc. ACM Symposium on User Interface Software and Technology, pp. 8190 (2006)

8. Wilson, G.: An analysis of mental workload in pilots during flight using multiple psychophysiological measures. International Journal of Aviation Psychology 12, 3-18 (2002)

9. Zarjam, P., Epps, J., Chen, F.: Evaluation of working memory load using EEG signals. In: Proc. APSIPA Annual Summit and Conference, pp. 715-719 (2010)

10. http://www.emotiv.com/ 